\title{
Randomised placebo-controlled trials of individualised homeopathic treatment: systematic review and meta-analysis
}

\author{
Robert T Mathie ${ }^{1 *}$, Suzanne M Lloyd² ${ }^{2}$ Lynn A Legg ${ }^{3}$, Jürgen Clausen, Sian Moss ${ }^{5}$, Jonathan RT Davidson ${ }^{6}$ \\ and lan Ford ${ }^{2}$
}

\begin{abstract}
Background: A rigorous and focused systematic review and meta-analysis of randomised controlled trials (RCTs) of individualised homeopathic treatment has not previously been undertaken. We tested the hypothesis that the outcome of an individualised homeopathic treatment approach using homeopathic medicines is distinguishable from that of placebos.

Methods: The review's methods, including literature search strategy, data extraction, assessment of risk of bias and statistical analysis, were strictly protocol-based. Judgment in seven assessment domains enabled a trial's risk of bias to be designated as low, unclear or high. A trial was judged to comprise 'reliable evidence' if its risk of bias was low or was unclear in one specified domain. 'Effect size' was reported as odds ratio (OR), with arithmetic transformation for continuous data carried out as required; OR > 1 signified an effect favouring homeopathy.
\end{abstract}

Results: Thirty-two eligible RCTs studied 24 different medical conditions in total. Twelve trials were classed 'uncertain risk of bias', three of which displayed relatively minor uncertainty and were designated reliable evidence; 20 trials were classed 'high risk of bias'. Twenty-two trials had extractable data and were subjected to meta-analysis; $\mathrm{OR}=1.53$ (95\% confidence interval (Cl) 1.22 to 1.91). For the three trials with reliable evidence, sensitivity analysis revealed $\mathrm{OR}=1.98$ (95\% Cl 1.16 to 3.38$)$.

Conclusions: Medicines prescribed in individualised homeopathy may have small, specific treatment effects. Findings are consistent with sub-group data available in a previous 'global' systematic review. The low or unclear overall quality of the evidence prompts caution in interpreting the findings. New high-quality RCT research is necessary to enable more decisive interpretation.

Keywords: Individualised homeopathy, Meta-analysis, Randomised controlled trials, Systematic review

\section{Background}

The nature of the research evidence in homeopathy is a matter of ongoing scientific debate. Homeopathy's advocates tend to deny the worth of randomised controlled trials (RCTs) [1] or over-interpret their findings, whilst its critics dispute the therapy's scientific rationale and the existence of any positive findings in the research literature [2]. There is a need to temper these divergent opinions by considering the existing RCT evidence from an objective, rigorous and transparent assessment of the

\footnotetext{
* Correspondence: rmathie@britishhomeopathic.org

'British Homeopathic Association, Luton, UK

Full list of author information is available at the end of the article
}

research, reflecting its particular nature and intrinsic methodological quality.

Five systematic reviews have examined the RCT research literature on homeopathy as a whole, including the broad spectrum of medical conditions that have been researched and by all forms of homeopathy: four of these 'global' systematic reviews reached the conclusion that, with important caveats [3], the homeopathic intervention probably differs from placebo [4-7]. By contrast, the most recent global systematic review, by Shang et al., concluded there was "weak evidence for a specific effect of homeopathic remedies...compatible with the notion 
that the clinical effects of homeopathy are placebo effects" [8].

Four of the above reviews have distinguished RCTs of individualised homeopathy, either by mere identification $[4,8]$ or in formal sub-group analysis [6,7]. In their overarching approaches, however, each of these five reviews has assessed together the RCT findings of all forms of homeopathy (individualised homeopathy, clinical homeopathy, complex homeopathy, isopathy) as if they are the same intervention. There are important differences between these therapeutic approaches, especially that individualised homeopathy typically involves a long interview between the practitioner and the patient, whereas the other three forms (non-individualised homeopathy) do not. For a placebo-controlled trial of individualised homeopathy, conclusions about 'efficacy' (specific effects) apply potentially to each or just some of the homeopathic medicines prescribed to the individual participants in that trial. A meta-analysis of such RCTs (including those with crossover design, which we excluded-see 'Methods') was published in 1998 [9], using methods that predated the current rigorous standards for conducting risk-of-bias assessments and sensitivity analysis: it reported a significant overall treatment effect that marginally was not sustained for the best-quality trials.

We aimed to clarify the results and inferences from RCTs of individualised homeopathy by conducting an up-to-date systematic review and meta-analysis to test the hypothesis: In the context of an RCT, and for the broad spectrum of medical conditions that have been researched, the main outcome of an individualised homeopathic treatment approach using homeopathic medicines is distinguishable from that of the same approach using placebos (i.e. individually prescribed homeopathic medicines have specific effects).

\section{Methods}

Methods comply with the PRISMA 2009 Checklist (see Additional file 1) and with our previously published protocol [10].

\section{Search strategy, data sources and trial eligibility}

A detailed description of the search methods used in this study has previously been published [11]. We conducted a systematic literature search to identify RCTs that compared individualised homeopathy with placebos, and for any medical condition. Each of the following electronic databases was searched from its inception up to the end of 2011, with a supplementary search of the same databases up to the end of 2013: AMED, CAM-Quest ${ }^{\circ}$, CINAHL, Cochrane Central Register of Controlled Trials, Embase, Hom-Inform, LILACS, PubMed, Science Citation Index and Scopus. For the 2012/13 update, CORE-
$\mathrm{Hom}^{\circ}$ was searched in addition, using the term 'randomised,' 'quasi-randomised' or 'unknown' in the 'Sequence generation' field.

The full electronic search strategy for PubMed (Cochrane Highly Sensitive Search Strategy) is given in our previous paper [11]: '((homeopath* OR homoeopath*) AND ((randomized controlled trial [pt]) OR (controlled clinical trial [pt]) OR (randomized [tiab]) OR (placebo [tiab]) OR (clinical trials as topic [mesh:noexp]) OR (randomly [tiab]) OR (trial [ti]))) NOT (animals [mh] NOT humans [mh])'.

As stated in our published protocol [10], we then excluded trials of crossover design, of radionically prepared homeopathic medicines, of homeopathic prophylaxis, of homeopathy combined with other (complementary or conventional) intervention, or for other specified reasons. The final explicit exclusion criterion was that the trial's team members-specifically the homeopathic practitioner/s-were not blinded to the assigned intervention. All remaining trials were eligible for systematic review.

\section{Outcome definitions}

We identified one 'main outcome measure' from each study using a refinement of the approaches adopted by Linde et al. and by Shang et al. $[6,8]$. Our selection of each trial's 'main outcome measure' at the study endpoint (i.e. at the end of scheduled follow-up, unless otherwise indicated) was based on a pre-specified hierarchical list in order of greatest to least importance, recommended by the World Health Organization (WHO) [12]. The WHO approach is an internationally accepted method to ensure that a selected outcome is the most vital to the functioning and health of the patient: it thus ensured our consistent selection of the most important and objective outcome per trial.

\section{Data extraction}

Two reviewers (RTM and either JC, JRTD, LAL or SM) independently identified the 'main outcome measure' and extracted data for each trial using a standard recording approach [10]. Discrepancies in the identification and interpretation of data were discussed and resolved by consensus.

\section{Assessment of risk of bias}

We used the domains of assessment as per the Cochrane risk-of-bias appraisal tool [13]. The extracted information enabled appraisal of freedom from risk of bias: 'Yes' (low risk), 'Unclear' (uncertain risk) or 'No' (high risk). We applied this approach to each of seven domains: sequence generation (domain I), allocation concealment used to implement the random sequence (II), blinding of participants and study personnel (IIIa), blinding of outcome assessors (IIIb), incomplete outcome data (IV), 
selective outcome reporting (V), and other sources of bias (VI). The source of any research sponsorship (i.e. potential for vested interest) was taken into account for sub-group analysis (see below), but not in risk-of-bias assessment per se.

Reflecting appropriately the designated 'main outcome measure, we rated risk of bias for each trial across all seven domains and using the following classification [10]:

Rating $\mathrm{A}=$ Low risk of bias in all seven domains.

Rating $\mathrm{B} x=$ Uncertain risk of bias in $x$ domains; low risk of bias in all other domains.

Rating Cy.x = High risk of bias in $y$ domains; uncertain risk of bias in $x$ domains; low risk of bias in all other domains.

\section{Designating an RCT as 'reliable evidence'}

An 'A'-rated trial comprises reliable evidence. We designated a 'B1'-rated trial reliable evidence if the uncertainty in its risk of bias was for one of domains IV, V or VI only (i.e. it was required to be judged free of bias for each of domains I, II, IIIa and IIIb).

\section{Statistical analysis \\ Data}

Mean, standard deviation and number of subjects were extracted for each continuous 'main outcome' and the standardised mean difference (SMD) calculated, reflecting whether a higher or a lower score was in the direction of the hypothesis favouring homeopathy; the number of favourable events and number of subjects were extracted for each dichotomous 'main outcome' and the odds ratio (OR) calculated. We did not adjust values to compensate for any inter-group differences at baseline.

For key meta-analyses, a single measure of 'effect size' was required to enable pooling of all relevant trials, and so SMD was transformed to OR using a recognised approximation method [10]. Random-effects meta-analysis models were used from the outset due to the known clinical heterogeneity among studies.

\section{Study selection for meta-analysis}

All RCTs that were included in the systematic review were potentially eligible for meta-analysis. If the original RCT paper did not provide or inform adequate information on our selected 'main outcome' to enable calculation of the SMD or the OR, we excluded the trial from meta-analysis and described the outcome as 'not estimable'.

\section{Heterogeneity and publication bias}

The $I^{2}$ statistic was used to assess the variability between studies; this statistic can take values between $0 \%$ and $100 \%$, with high values indicative of strong heterogeneity.
Funnel plots and Egger's test were used to assess publication bias $[14,15]$.

\section{Sensitivity and sub-group analysis}

Sensitivity and sub-group analyses were carried out, consistent with our published protocol [10]. The sensitivity analysis examined the impact on the pooled OR of trials' risk-of-bias ratings. Included in sub-group analyses, we aimed to examine whether a trial's homeopathic medicines had potency $\geq 12 \mathrm{C}$ or $<12 \mathrm{C}$ (12-time serial dilution of 1:100 starting solution), a concentration sometimes regarded as equivalent to the 'Avogadro limit' for molecular dose [16].

\section{Results}

Included studies

The PRISMA flowchart from the original comprehensive literature search has been published previously [11] and comprises 489 records. The corresponding flowchart for RCT records published in 2012/13 is given in Additional file 2, which features 44 new records. A composite PRISMA flowchart, in standardised format, is given in Additional file 3.

Our updated literature search identified a total of five new records that were potentially eligible for the current review of RCTs that compared individualised homeopathy with placebos (Additional file 4, an update of the flowchart in our published protocol [10]). All five were single-blinded and thus ineligible for systematic review.

In total, therefore, 31 records fulfilled the relevant inclusion criteria. Data were non-extractable from 10 of those (see Additional file 4), leaving 21 records potentially available for meta-analysis. One of the included records reported findings from two RCTs. Thus, the systematic review comprises 32 RCTs, with metaanalysis of 22 of those RCTs.

\section{Demographic data}

The 32 RCTs represented 24 different medical conditions across 12 categories (Table 1). Homeopathic potency was $\geq 12 \mathrm{C}$ in 12 trials and was not exclusively $\geq 12 \mathrm{C}$ for 20 trials (mix of $>12 \mathrm{C}$ and $<12 \mathrm{C}$ for 12 trials; unstated for 8 trials). Vested interest was absent in four trials, uncertain in 18 and present in 10.

\section{Summary of findings}

For each trial, Table 2 includes details of the sample size, the identified 'main outcome measure' (and whether dichotomous or continuous), the end-point and whether the study was described in the original report as a 'pilot' (or 'preliminary' or 'feasibility') study. The median sample size (for $N=32$ trials) was 43.5 (inter-quartile range 27 to 67 ). There were 28 different 'main outcome 
Table 1 Demographic data for 32 randomised controlled trials (RCTs) of individualised homeopathy

\begin{tabular}{|c|c|c|c|c|c|c|c|c|c|}
\hline \# & First author & Year & Category & Condition & Participants' demographics & Study setting & $\begin{array}{l}\text { Potency } \\
\geq 12 C / 24 D\end{array}$ & Funding source & $\begin{array}{l}\text { Free of } \\
\text { vested } \\
\text { interest }^{\mathrm{a}}\end{array}$ \\
\hline A01 & Andrade & 1991 & Rheumatology & $\begin{array}{l}\text { Rheumatoid } \\
\text { arthritis }\end{array}$ & $\begin{array}{l}\text { Patients with active RA and } \\
\text { fulfilling at least three } \\
\text { pre-defined criteria }\end{array}$ & $\begin{array}{c}\text { Rheumatic disease } \\
\text { outpatient clinic, Escola } \\
\text { Paulista de Medicina, São } \\
\text { Paulo, Brazil }\end{array}$ & Mixed & None stated & U \\
\hline A05 & Bell & 2004 & Rheumatology & Fibromyalgia & Fibromyalgia patients & Private clinic in the USA & Mixed & External (government) & Y \\
\hline A06 & Bonne & 2003 & Mental disorder & Anxiety & $\begin{array}{l}\text { Adults aged 18-65 years, of either sex, } \\
\text { suffering from anxiety as defined by } \\
\text { standard psychometric criteria }\end{array}$ & $\begin{array}{l}\text { Department of Psychiatry, } \\
\text { Hadassah University } \\
\text { Hospital, Jerusalem, Israel }\end{array}$ & Y & None stated & U \\
\hline A07 & Brien & 2011 & Rheumatology & $\begin{array}{l}\text { Rheumatoid } \\
\text { arthritis }\end{array}$ & $\begin{array}{l}\text { Adults formally diagnosed with } \\
\text { RA for at least } 2 \text { years, who had } \\
\text { relatively stable disease but some } \\
\text { disease activity on entry }\end{array}$ & $\begin{array}{l}\text { Rheumatology outpatient } \\
\text { departments at three } \\
\text { hospitals, UK }\end{array}$ & U & $\begin{array}{c}\text { National Institute of Health Research; } \\
\text { Samueli Institute, USA; Southampton } \\
\text { Complementary Medicine Research } \\
\text { Trust; Rufford Maurice Laing } \\
\text { Foundation; Dreluso Pharmazeutika } \\
\text { GmBH; National Health Service Fund for } \\
\text { Science }\end{array}$ & Y \\
\hline A09 & Cavalcanti & 2003 & Dermatology & $\begin{array}{l}\text { Uraemic } \\
\text { pruritus }\end{array}$ & $\begin{array}{l}\text { Dialysis for more than } 6 \text { months, } \\
\text { moderate to severe pruritus, } \\
\text { absence of other causes for pruritus }\end{array}$ & $\begin{array}{l}\text { Haemodialysis centres, Rio } \\
\text { de Janeiro state, Brazil }\end{array}$ & Mixed & None stated & U \\
\hline A10 & Chapman & 1999 & Neurology & Brain injury & Patients with mild traumatic brain injury & $\begin{array}{l}\text { University medical school } \\
\text { in the USA }\end{array}$ & Y & $\begin{array}{l}\text { External (government; foundation; hom } \\
\text { pharm providing all meds) }\end{array}$ & N \\
\hline A11 & $\begin{array}{l}\text { de Lange de } \\
\text { Klerk }\end{array}$ & 1994 & $\begin{array}{l}\text { Respiratory } \\
\text { infection }\end{array}$ & URTI & $\begin{array}{l}\text { Children aged } 1.5-10 \text { years, with at least } \\
\text { three upper respiratory tract infections in } \\
\text { the past year or with two such episodes } \\
\text { plus otitis media with effusion at the } \\
\text { time of entry examination }\end{array}$ & $\begin{array}{l}\text { Paediatric outpatient } \\
\text { department of university } \\
\text { hospital, Amsterdam, } \\
\text { Netherlands }\end{array}$ & Mixed & $\begin{array}{l}\text { Dutch Ministry of Welfare, Cultural } \\
\text { Affairs, and Public Health }\end{array}$ & Y \\
\hline A13 & Fisher & 2006 & Dermatology & Eczema & $\begin{array}{l}\text { Adults aged 18-65 years, of either sex, } \\
\text { with planned treatment for } \\
\text { dermatitis at the RLHH }\end{array}$ & $\begin{array}{l}\text { Royal London } \\
\text { Homoeopathic Hospital } \\
\text { (RLHH), London, UK }\end{array}$ & Mixed & None stated & U \\
\hline A14 & Frass & 2005 & $\begin{array}{l}\text { Surgery and } \\
\text { anaesthesiology }\end{array}$ & Sepsis & Patients with severe sepsis & $\begin{array}{l}\text { Intensive Care Unit at a } \\
\text { university hospital in } \\
\text { Austria }\end{array}$ & Y & None stated & U \\
\hline A16 & Gaucher & 1994 & Tropical disease & Cholera & $\begin{array}{l}\text { Patients with cholera, in a } \\
\text { state of dehydration requiring } \\
\text { parenteral treatment }\end{array}$ & $\begin{array}{l}\text { University of San Marcos, } \\
\text { Lima, Peru }\end{array}$ & U & $\begin{array}{c}\text { None stated; several acknowledged, } \\
\text { including employee of hom pharm } \\
\text { company }\end{array}$ & $u$ \\
\hline A18 & Jacobs & 1993 & Gastroenterology & $\begin{array}{l}\text { Childhood } \\
\text { diarrhoea }\end{array}$ & $\begin{array}{l}\text { Children aged } 0.5-5 \text { years, with } \\
\text { history of acute diarrhoea }\end{array}$ & $\begin{array}{l}\text { Participants' homes, Leon, } \\
\text { Nicaragua }\end{array}$ & Y & $\begin{array}{c}\text { Boiron Research Foundation, Norwood, } \\
\text { Pennsylvania, USA, }\end{array}$ & U \\
\hline A19 & Jacobs & 1994 & Gastroenterology & $\begin{array}{l}\text { Childhood } \\
\text { diarrhoea }\end{array}$ & Children with a history of acute diarrhoea & Nicaragua & Y & $\begin{array}{c}\text { External, including hom pharm that } \\
\text { might have provided the remedies for } \\
\text { the trial }\end{array}$ & u \\
\hline A20 & Jacobs & 2001 & $\begin{array}{l}\text { Ear, nose and } \\
\text { throat }\end{array}$ & $\begin{array}{l}\text { Otitis media } \\
\quad \text { (acute) }\end{array}$ & Patients with acute otitis media & Children's clinic in the USA & Y & $\begin{array}{l}\text { External (hom pharm—all } \\
\text { remedies—research grant) }\end{array}$ & N \\
\hline
\end{tabular}


Table 1 Demographic data for 32 randomised controlled trials (RCTs) of individualised homeopathy (Continued)

\begin{tabular}{|c|c|c|c|c|c|c|c|c|c|}
\hline A21 & Jacobs & 2000 & Gastroenterology & $\begin{array}{l}\text { Childhood } \\
\text { diarrhoea }\end{array}$ & $\begin{array}{l}\text { Children with a history } \\
\text { of acute diarrhoea }\end{array}$ & Nepal & Y & External (hom research foundation) & U \\
\hline A22 & Jacobs & $2005 b$ & $\begin{array}{l}\text { Obstetrics and } \\
\text { gynaecology }\end{array}$ & $\begin{array}{l}\text { Menopause } \\
\text { post breast } \\
\text { cancer }\end{array}$ & $\begin{array}{l}\text { Women with history of carcinoma or } \\
\text { stage I-III breast cancer who had } \\
\text { completed all surgery, chemotherapy and } \\
\text { radiation treatment and who averaged at } \\
\text { least three hot flushes per day for } \\
\text { previous month }\end{array}$ & $\begin{array}{l}\text { Private medical clinic, } \\
\text { Seattle, USA }\end{array}$ & U & $\begin{array}{l}\text { External (charity); meds donated by } \\
\text { hom pharm }\end{array}$ & N \\
\hline A23 & Jacobs & $2005 a$ & Mental disorder & ADHD & $\begin{array}{c}\text { Children, aged } 6-12 \text { years, meeting } \\
\text { DSM-IV criteria for ADHD }\end{array}$ & $\begin{array}{l}\text { Private homeopathic clinic } \\
\text { in Seattle, USA }\end{array}$ & U & $\begin{array}{l}\text { External (government); meds donated } \\
\text { by hom pharm }\end{array}$ & $\mathrm{N}$ \\
\hline A24 & Jansen & 1992 & Gastroenterology & Proctocolitis & $\begin{array}{l}\text { Active proctocolitis (proven by } \\
\text { endoscopy and histology) }\end{array}$ & $\begin{array}{l}\text { Outpatient dept, Maria } \\
\text { Hospital, Tilburg, } \\
\text { Netherlands }\end{array}$ & Y & None stated & $U$ \\
\hline A25 & Kainz & 1996 & Dermatology & Warts & $\begin{array}{c}\text { Children aged 6-12 years, with common } \\
\text { warts on the back of hands }\end{array}$ & $\begin{array}{l}\text { Department of } \\
\text { Dermatology, University of } \\
\text { Graz, Austria }\end{array}$ & Mixed & $\begin{array}{l}\text { Firma Spagyra, Groding, Austria: 'kindly } \\
\text { provided' homeopathic medicines and } \\
\text { placebos. Otherwise, none stated }\end{array}$ & $\mathrm{N}$ \\
\hline A26 & Katz & 2005 & Mental disorder & Depression & $\begin{array}{l}\text { Adults, aged } 18-80 \text { years, of either sex, } \\
\text { suffering from major depressive episode } \\
\text { of moderate severity as defined by DSM-IV }\end{array}$ & $\begin{array}{l}\text { Lower Clapton group } \\
\text { practice, east London, UK }\end{array}$ & U & $\begin{array}{l}\text { Homeopathic Research Committee; } \\
\text { Blackie Foundation Trust. Verum and } \\
\text { placebo homeopathic medicines } \\
\text { donated by Laboratoires Boiron }\end{array}$ & N \\
\hline A30 & Naudé & 2010 & Mental disorder & Insomnia & $\begin{array}{l}\text { Insomnia sufferers (identified through } \\
\text { local advertising) }\end{array}$ & South Africa & Mixed & None stated & U \\
\hline A31 & Rastogi (a) & 1999 & $\begin{array}{l}\text { Immune } \\
\text { disorder }\end{array}$ & HIV & $\begin{array}{c}\text { Adults aged } 18-50 \text { years, of either sex, } \\
\text { with positive antibody reaction to HIV-1 or } \\
\text { HIV-2 or both confirmed by repeat ELISA } \\
\text { and/or Western blot }\end{array}$ & $\begin{array}{l}\text { Regional Research Institute } \\
\text { for Homoeopathy, } \\
\text { Mumbai, India }\end{array}$ & Mixed & None stated & $U$ \\
\hline A31 & Rastogi (b) & 1999 & $\begin{array}{l}\text { Immune } \\
\text { disorder }\end{array}$ & HIV & $\begin{array}{c}\text { Adults aged } 18-50 \text { years, of either sex, } \\
\text { with positive antibody reaction to HIV-1 or } \\
\text { HIV-2 or both confirmed by repeat ELISA } \\
\text { and/or Western blot }\end{array}$ & $\begin{array}{l}\text { Regional Research Institute } \\
\text { for Homoeopathy, } \\
\text { Mumbai, India }\end{array}$ & Mixed & None stated & U \\
\hline A32 & Sajedi & 2008 & Neurology & $\begin{array}{l}\text { Cerebral } \\
\text { palsy }\end{array}$ & $\begin{array}{l}\text { Children aged } 1-5 \text { years, with mild to } \\
\text { moderate spasm due to cerebral palsy }\end{array}$ & $\begin{array}{l}\text { Saba Clinic } \\
\text { (Developmental Disorder } \\
\text { Center), Tehran, Iran }\end{array}$ & U & Internal & U \\
\hline A33 & Siebenwirth & 2009 & Dermatology & Eczema & $\begin{array}{l}\text { Adults aged } 18-35 \text { years with at } \\
\text { least a 1-year history of atopic } \\
\text { dermatitis ( }>20 \% \text { of skin surface) }\end{array}$ & $\begin{array}{l}\text { Klinik und poliklinik für } \\
\text { Dermatologie und } \\
\text { Allergologie am } \\
\text { Biederstein, Munich, } \\
\text { Germany }\end{array}$ & Mixed & $\begin{array}{l}\text { The study medications gifted by } \\
\text { Deutsche Homöopathie-Union (DHU). } \\
\text { Funded by Karl und Veronica Carstens- } \\
\text { Stiftung }\end{array}$ & $\mathrm{N}$ \\
\hline A34 & Steinsbekk & 2005 & $\begin{array}{l}\text { Respiratory } \\
\text { infection }\end{array}$ & URTI & $\begin{array}{l}\text { Children, under } 10 \text { years old, who had } \\
\text { been to a medical doctor for URTI }\end{array}$ & $\begin{array}{l}\text { In children's own homes, } \\
\text { recruited mainly from } \\
\text { those previously } \\
\text { diagnosed with URTI } \\
\text { when attending a hospital } \\
\text { casualty department }\end{array}$ & Y & $\begin{array}{c}\text { External: Norwegian Research Council. } \\
\text { Homeoden Belgium made the } \\
\text { medicines }\end{array}$ & U \\
\hline
\end{tabular}


Table 1 Demographic data for 32 randomised controlled trials (RCTs) of individualised homeopathy (Continued)

\begin{tabular}{|c|c|c|c|c|c|c|c|c|c|}
\hline A35 & Straumsheim & 2000 & Neurology & Migraine & $\begin{array}{l}\text { Adults aged 18-65 years, of either sex, } \\
\text { diagnosed according to International } \\
\text { Headache Society classification criteria }\end{array}$ & $\begin{array}{l}\text { Arena Medisinske Senter, } \\
\text { Oslo, Norway }\end{array}$ & Mixed & $\begin{array}{l}\text { DCG Farmaceutia (Gotenborg): supplied } \\
\text { all homeopathic medicines. Norwegian } \\
\text { Research Council }\end{array}$ & N \\
\hline A36 & Thompson & 2005 & $\begin{array}{l}\text { Obstetrics and } \\
\text { gynaecology }\end{array}$ & $\begin{array}{l}\text { Menopause } \\
\text { post breast } \\
\text { cancer }\end{array}$ & $\begin{array}{l}\text { Women who attended oncology } \\
\text { centre for breast cancer, did not have } \\
\text { metastatic disease, were not on any } \\
\text { other treatment and experiencing } \\
\text { more than three hot flushes per day }\end{array}$ & $\begin{array}{l}\text { Outpatient department of } \\
\text { an NHS homeopathic } \\
\text { hospital, UK }\end{array}$ & Y & $\begin{array}{l}\text { External (charity; hom pharm } \\
\text { —'provided' all remedies, presumably } \\
\text { via hospital pharmacy dept) }\end{array}$ & $U$ \\
\hline A37 & Walach & 1997 & Neurology & Headache & $\begin{array}{l}\text { Patients diagnosed according to } \\
\text { the International Headache } \\
\text { Society classification criteria }\end{array}$ & Germany & Mixed & $\begin{array}{c}\text { Homeoden (Gent) and Gudjons } \\
\text { (Augsburg): supplied homeopathic } \\
\text { medicines }\end{array}$ & $U$ \\
\hline A38 & $\begin{array}{l}\text { Weatherley- } \\
\text { Jones }\end{array}$ & 2004 & Mental disorder & $\begin{array}{l}\text { Chronic } \\
\text { fatigue } \\
\text { syndrome }\end{array}$ & Adults with chronic fatigue & $\begin{array}{l}\text { Two outpatient } \\
\text { departments in UK }\end{array}$ & $U$ & External (charity) & Y \\
\hline A39 & White & 2003 & $\begin{array}{l}\text { Allergy and } \\
\text { asthma }\end{array}$ & $\begin{array}{l}\text { Childhood } \\
\text { asthma }\end{array}$ & $\begin{array}{l}\text { Children, aged 5-15 years, with diagnosis } \\
\text { of asthma and prescription for beta } \\
\text { agonist and/or corticosteroid inhaler } \\
\text { issued within previous } 3 \text { months }\end{array}$ & $\begin{array}{c}\text { Five general practices in } \\
\text { market towns in Somerset, } \\
\text { UK }\end{array}$ & U & $\begin{array}{l}\text { External, including hom pharm that } \\
\text { provided all remedies }\end{array}$ & N \\
\hline A40 & Whitmarsh & 1997 & Neurology & Migraine & $\begin{array}{l}\text { Adults aged } 18-60 \text { years, of either sex, } \\
\text { with definite diagnosis of migraine } \\
\text { by pre-defined criteria }\end{array}$ & $\begin{array}{l}\text { Princess Margaret Migraine } \\
\text { Clinic, Charing Cross } \\
\text { Hospital, London, UK }\end{array}$ & Y & $\begin{array}{c}\text { Nelsons Ltd; Homeopathic Medical } \\
\text { Research Council; Blackie Foundation } \\
\text { Trust }\end{array}$ & U \\
\hline A41 & Yakir & 2001 & $\begin{array}{l}\text { Obstetrics and } \\
\text { gynaecology }\end{array}$ & $\begin{array}{l}\text { Premenstrual } \\
\text { syndrome }\end{array}$ & $\begin{array}{l}\text { Women aged } 20-50 \text { years, with diagnosis } \\
\text { of PMS according to pre-defined criteria; } \\
\text { symptomatology corresponding to one of } \\
\text { five homeopathic medicines }\end{array}$ & $\begin{array}{l}\text { Gynaecology outpatient } \\
\text { clinic, Hadassah University } \\
\text { Hospital, Jerusalem, Israel }\end{array}$ & Y & $\begin{array}{l}\text { Dolisos Laboratories; Deutsche } \\
\text { Homeopathie Union; named individuals }\end{array}$ & N \\
\hline
\end{tabular}

research sponsorship; indirect, via gifted medicines) from a company that provided homeopathic medicines for the trial. 
Table 2 Summary of findings table

\begin{tabular}{|c|c|c|c|c|c|c|c|c|c|c|}
\hline$\#$ & First author & Year & Pilot & $\begin{array}{l}\text { ITT } \\
\text { sample }\end{array}$ & $\begin{array}{c}\text { PP } \\
\text { sample }\end{array}$ & $\begin{array}{l}\text { PP sample }> \\
\text { median (43.5) }\end{array}$ & $\begin{array}{l}\text { Attrition } \\
\text { rate(\%) }\end{array}$ & ‘Main' outcome identified & $\begin{array}{l}\text { Nature of 'main' } \\
\text { outcome }\end{array}$ & End-point \\
\hline $\mathrm{A} 01$ & Andrade & 1991 & $\mathrm{~N}$ & 44 & 33 & $\mathrm{~N}$ & 25.0 & Ritchie articular index & Continuous & 6 months \\
\hline A05 & Bell & 2004 & Y & 62 & 53 & Y & 14.5 & Tender point pain on palpation & Continuous & 3 months \\
\hline A06 & Bonne & 2003 & N & 44 & 39 & $\mathrm{~N}$ & 11.4 & Hamilton Rating Scale for Anxiety (HAM-A) & Continuous & 10 weeks \\
\hline A07 & Brien & 2011 & N & 32 & 23 & $\mathrm{~N}$ & 28.1 & $\begin{array}{l}\text { Proportion of patients meeting ACR } 20 \% \\
\text { improvement criteria ('ACR20 response') }\end{array}$ & Dichotomous & 28 weeks \\
\hline A09 & Cavalcanti & 2003 & N & 28 & 20 & N & 28.6 & $\begin{array}{l}\text { Responders: patients with more than } 50 \% \\
\text { reduction in pruritus score }\end{array}$ & Dichotomous & 60 days \\
\hline $\mathrm{A} 10$ & Chapman & 1999 & Y & 61 & 50 & Y & 18.0 & $\begin{array}{l}\text { SRH-SLPD functional assessment tool: } \\
\text { SRS (symptoms) sub-scale }\end{array}$ & Continuous & 4 months \\
\hline A11 & $\begin{array}{l}\text { de Lange de } \\
\text { Klerk }\end{array}$ & 1994 & N & 175 & 170 & Y & 2.9 & Daily total symptom score & Continuous & 1 year \\
\hline $\mathrm{A} 13$ & Fisher & 2006 & Y & 38 & 27 & N & 28.9 & VAS of overall symptom severity & Continuous & 13 weeks \\
\hline A14 & Frass & 2005 & N & 70 & 67 & Y & 4.3 & Patient survival & Dichotomous & 180 days \\
\hline A16 & Gaucher & 1994 & N & 80 & 51 & Y & 36.3 & Degree of dehydration & Continuous & $12 \mathrm{~h}$ \\
\hline A18 & Jacobs & 1993 & N & 34 & 33 & $\mathrm{~N}$ & 2.9 & $\begin{array}{c}\text { Number of days until fewer than } 3 \text { unformed } \\
\text { stools for } 2 \text { consecutive days }\end{array}$ & Continuous & Up to 6 days \\
\hline A19 & Jacobs & 1994 & N & 92 & 81 & Y & 12.0 & $\begin{array}{l}\text { Number of days until fewer than } 3 \text { unformed } \\
\text { stools for } 2 \text { consecutive days }\end{array}$ & Continuous & Up to 5 days \\
\hline A20 & Jacobs & 2001 & Y & 75 & 75 & Y & 0.0 & Treatment failure & Dichotomous & 5 days (cumulative total) \\
\hline A21 & Jacobs & 2000 & N & 126 & 116 & Y & 7.9 & $\begin{array}{l}\text { Number of days until fewer than } 3 \text { unformed } \\
\text { stools for } 2 \text { consecutive days }\end{array}$ & Continuous & 5 days \\
\hline A22 & Jacobs & $2005 b$ & Y & 53 & 33 & N & 37.7 & Hot flash severity score & Continuous & 12 months \\
\hline A23 & Jacobs & $2005 a$ & Y & 43 & 37 & $\mathrm{~N}$ & 14.0 & Conners Global Index-Parent (CGI-P)_-total & Continuous & 17 weeks \\
\hline A24 & Jansen & 1992 & Y & 10 & 4 & $\mathrm{~N}$ & 60.0 & Endoscopic appearance (grade) & Continuous & 12 months \\
\hline A25 & Kainz & 1996 & N & 67 & 60 & Y & 10.4 & $\begin{array}{l}\text { Responders: patients with at least } 50 \% \\
\text { reduction in area of skin affected by warts }\end{array}$ & Dichotomous & 8 weeks \\
\hline A26 & Katz & 2005 & Y & 7 & 3 & $\mathrm{~N}$ & 57.1 & Hamilton Depression Scale (HAMD) & Continuous & 12 weeks \\
\hline $\mathrm{A} 30$ & Naudé & 2010 & N & 33 & 30 & N & 9.1 & Sleep Impairment Index (SII) summary score & Continuous & 4 weeks \\
\hline A31 & Rastogi (a) & 1999 & N & 50 & 42 & N & 16.0 & CD4+ T-lymphocyte counts & Continuous & 6 months \\
\hline A31 & Rastogi (b) & 1999 & N & 50 & 38 & $\mathrm{~N}$ & 24.0 & CD4+ T-lymphocyte counts & Continuous & 6 months \\
\hline A32 & Sajedi & 2008 & N & 24 & 16 & $\mathrm{~N}$ & 33.3 & $\begin{array}{l}\text { Modified Ashworth Scale: measurement of } \\
\text { muscle tone in right leg }\end{array}$ & Continuous & 4 months \\
\hline A33 & Siebenwirth & 2009 & N & 24 & 14 & $\mathrm{~N}$ & 41.7 & MP (multiparameter dermatitis) score & Continuous & 32 weeks \\
\hline A34 & Steinsbekk & 2005 & N & 251 & 199 & Y & 20.7 & Parent-reported URTI total symptom score & Continuous & Duration of 12 weeks \\
\hline A35 & Straumsheim & 2000 & $\mathrm{~N}$ & 73 & 68 & Y & 6.8 & Frequency of migraine attacks per month & Continuous & $\begin{array}{l}\text { Last month of 4-month } \\
\text { period }\end{array}$ \\
\hline
\end{tabular}


Table 2 Summary of findings table (Continued)

\begin{tabular}{|c|c|c|c|c|c|c|c|c|c|c|}
\hline $\mathrm{A} 36$ & Thompson & 2005 & $\mathrm{Y}$ & 53 & 45 & $\mathrm{Y}$ & 15.1 & MYMOP profile score & Continuous & 16 weeks \\
\hline A37 & Walach & 1997 & $\mathrm{~N}$ & 98 & 92 & Y & 6.1 & Frequency of headaches per month & Continuous & $\begin{array}{l}\text { Last month of 3-month } \\
\text { period }\end{array}$ \\
\hline A38 & Weatherley-Jones & 2004 & N & 103 & 86 & Y & 16.5 & $\begin{array}{l}\text { Responders: those with clinical } \\
\text { improvement (Multidimensional } \\
\text { Fatigue Inventory: mental fatigue) }\end{array}$ & Dichotomous & 7 months \\
\hline A39 & White & 2003 & N & 89 & 74 & Y & 16.9 & $\begin{array}{l}\text { Childhood Asthma Questionnaire (CAQ) } \\
\text { sub-scale for severity of symptoms }\end{array}$ & Continuous & 52 weeks \\
\hline A40 & Whitmarsh & 1997 & N & 63 & 60 & Y & 4.8 & Frequency of migraine attacks per month & Continuous & $\begin{array}{l}\text { Last month of 4-month } \\
\text { period }\end{array}$ \\
\hline A41 & Yakir & 2001 & Y & 23 & 19 & N & 17.4 & Menstrual distress questionnaire (MDQ) score & Continuous & $\begin{array}{l}\text { Duration of } 3 \text { months } \\
\text { (last } 7 \text { days per cycle) }\end{array}$ \\
\hline
\end{tabular}

$I T$ intention to treat, $P P$ per protocol, $Y$ yes, $N$ no. 
measures' and for an end-point that ranged from $12 \mathrm{~h}$ to 12 months.

\section{Risk of bias and reliable evidence}

Table 3 provides the risk-of-bias details for each of the 32 trials and sub-divided by (a) included in our meta- analysis and (b) not included in our meta-analysis. Domain IIIa (blinding of participants and trial personnel) contributed the least risk of bias overall, with no trial classed 'high risk'. Domain IV (completeness of outcome data) presented the greatest methodological concerns, with 14 trials judged 'high risk'. Domain II (allocation

Table 3 Risk-of-bias assessments for trials: (a) included in meta-analysis and (b) not included in meta-analysis

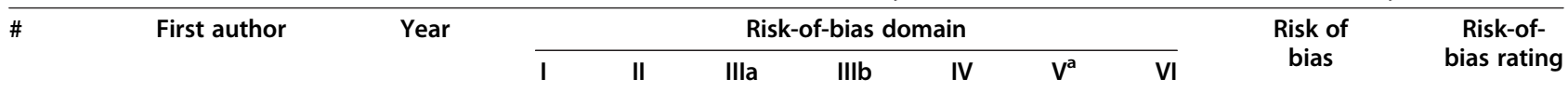

\section{(a): Included in meta-analysis}

\begin{tabular}{|c|c|c|c|c|c|c|c|c|c|c|c|}
\hline A11 & de Lange de Klerk & 1994 & U & $U$ & $U$ & U & Y & Y & Y & Uncertain & B4 \\
\hline A19 & Jacobs & 1994 & Y & Y & Y & Y & U & Y & Y & Uncertain $^{\text {b }}$ & B1 \\
\hline A25 & Kainz & 1996 & U & $U$ & U & $U$ & $U$ & Y & $U$ & Uncertain & B6 \\
\hline A10 & Chapman & 1999 & Y & U & Y & Y & Y & Y & Y & Uncertain & B1 \\
\hline A35 & Straumsheim & 2000 & U & U & Y & Y & Y & Y & Y & Uncertain & B2 \\
\hline A20 & Jacobs & 2001 & Y & Y & Y & Y & U & Y & Y & Uncertain $^{\mathrm{b}}$ & B1 \\
\hline A41 & Yakir & 2001 & U & U & Y & Y & U & Y & Y & Uncertain & B3 \\
\hline A06 & Bonne & 2003 & U & $U$ & Y & Y & Y & Y & $U$ & Uncertain & B3 \\
\hline A05 & Bell & 2004 & Y & Y & Y & Y & Y & Y & U & Uncertain $^{b}$ & B1 \\
\hline A14 & Frass & 2005 & Y & $U$ & Y & $U$ & Y & Y & Y & Uncertain & B2 \\
\hline A23 & Jacobs & $2005 a$ & Y & U & Y & Y & Y & Y & Y & Uncertain & B1 \\
\hline A36 & Thompson & 2005 & Y & $U$ & Y & Y & Y & Y & Y & Uncertain & B1 \\
\hline A40 & Whitmarsh & 1997 & U & $U$ & Y & U & Y & $U$ & $\mathrm{~N}$ & High & C1.4 \\
\hline A31 & Rastogi (a) & 1999 & U & U & U & U & $\mathrm{N}$ & $\mathrm{N}$ & U & High & $\mathrm{C} 2.5$ \\
\hline A31 & Rastogi (b) & 1999 & U & $U$ & U & U & $\mathrm{N}$ & $\mathrm{N}$ & $U$ & High & $\mathrm{C} 2.5$ \\
\hline A09 & Cavalcanti & 2003 & U & U & Y & Y & $\mathrm{N}$ & Y & $U$ & High & C1.3 \\
\hline A38 & Weatherley-Jones & 2004 & Y & $U$ & Y & Y & N & Y & Y & High & $\mathrm{C} 1.1$ \\
\hline A22 & Jacobs & $2005 b$ & Y & Y & Y & Y & $\mathrm{N}$ & Y & Y & High & C1.0 \\
\hline A13 & Fisher & 2006 & Y & U & Y & Y & N & Y & U & High & $\mathrm{C} 1.2$ \\
\hline A32 & Sajedi & 2008 & U & $U$ & $U$ & $U$ & $\mathrm{~N}$ & Y & Y & High & C1.4 \\
\hline A33 & Siebenwirth & 2009 & U & Y & Y & Y & N & Y & N & High & C2.1 \\
\hline A07 & Brien & 2011 & Y & Y & Y & Y & $\mathrm{N}$ & Y & Y & High & C1.0 \\
\hline \multicolumn{12}{|c|}{ (b): Not included in meta-analysis } \\
\hline A01 & Andrade & 1991 & U & $U$ & U & $U$ & $\mathrm{~N}$ & $\mathrm{~N}$ & U & High & $\mathrm{C} 2.5$ \\
\hline A24 & Jansen & 1992 & U & U & U & U & $\mathrm{N}$ & $\mathrm{N}$ & $\mathrm{N}$ & High & C 3.4 \\
\hline A18 & Jacobs & 1993 & U & U & Y & Y & U & $\mathrm{N}$ & U & High & C1.4 \\
\hline A16 & Gaucher & 1994 & N & $N$ & U & U & $\mathrm{N}$ & $\mathrm{N}$ & $U$ & High & $C 4.3$ \\
\hline A37 & Walach & 1997 & U & Y & Y & U & Y & $N$ & $N$ & High & $\mathrm{C} 2.2$ \\
\hline A21 & Jacobs & 2000 & Y & Y & Y & Y & Y & N & $\mathrm{N}$ & High & $\mathrm{C} 2.0$ \\
\hline A39 & White & 2003 & Y & Y & Y & Y & Y & $\mathrm{N}$ & Y & High & $\mathrm{C} 1.0$ \\
\hline A26 & Katz & 2005 & Y & $U$ & Y & U & $\mathrm{N}$ & $N$ & $\mathrm{~N}$ & High & C 3.2 \\
\hline A34 & Steinsbekk & 2005 & Y & Y & Y & Y & $\mathrm{N}$ & $N$ & Y & High & C2.0 \\
\hline A30 & Naudé & 2010 & Y & U & Y & Y & U & $\mathrm{N}$ & Y & High & $\mathrm{C} 1.2$ \\
\hline
\end{tabular}

Trials are arranged chronologically within their risk-of-bias rating category.

'Unless a published study protocol was available, completeness of reporting (domain V) was judged solely on correspondence of 'Results' with details in 'Methods' section of paper.

${ }^{\mathrm{b}}$ Reliable evidence.

$Y$ yes, $U$ unclear, $N$ no (regarding freedom from risk of bias). 
concealment) presented the most uncertain methodological judgments, with 21 (66\%) trials assessed 'unclear risk' and 10 (31\%) assessed 'low risk'. A risk-of-bias bar graph is shown in Additional file 5.

Table 3(a): No trial was 'A'-rated (low risk of bias overall)-i.e. none fulfilled the criteria for all seven domains of assessment. Table 3(a) therefore includes a list of 12 trials that were classed uncertain risk of bias ('B'-rated) and 10 that were classed high risk of bias ('C'-rated). Table 3(a) also shows the three 'B1'-rated trials that satisfied our criteria for reliable evidence. All other trials had unclear or high risk of bias in important methodological aspects and may be regarded as non-reliable evidence.

Table 3(b): Trials that were deficient in domain V (selective outcome reporting) included the ten whose data were not extractable for meta-analysis and which were thus ' $C$ '-rated by default; seven of these ten trials were already ' $C$ '-rated due to deficiency in at least one other domain of assessment.

\section{Meta-analysis}

The data extracted per trial (continuous or dichotomous data) are tabulated in Additional file 6. Figure 1 illustrates the OR for each trial; the original SMD data for each of the studies with continuous data are shown in Additional file 7 . Of the 22 trials, 15 had an effect favouring homeopathy (i.e. OR $>1$ ), 3 of them statistically significantly; 7 trials had an effect favouring placebo, none of them significantly. Total sample size = $1,123(N=22$ trials $)$.

Pooled OR was 1.53 (95\% confidence interval (CI) 1.22 to $1.91 ; P<0.001)$. There was no difference depending on whether the 'main outcome' was continuous (OR = 1.45 ; $95 \%$ CI 1.12 to 1.89 ) or dichotomous ( $\mathrm{OR}=1.80$ (95\% CI 1.12 to 2.87$) ; P=0.44$ ).

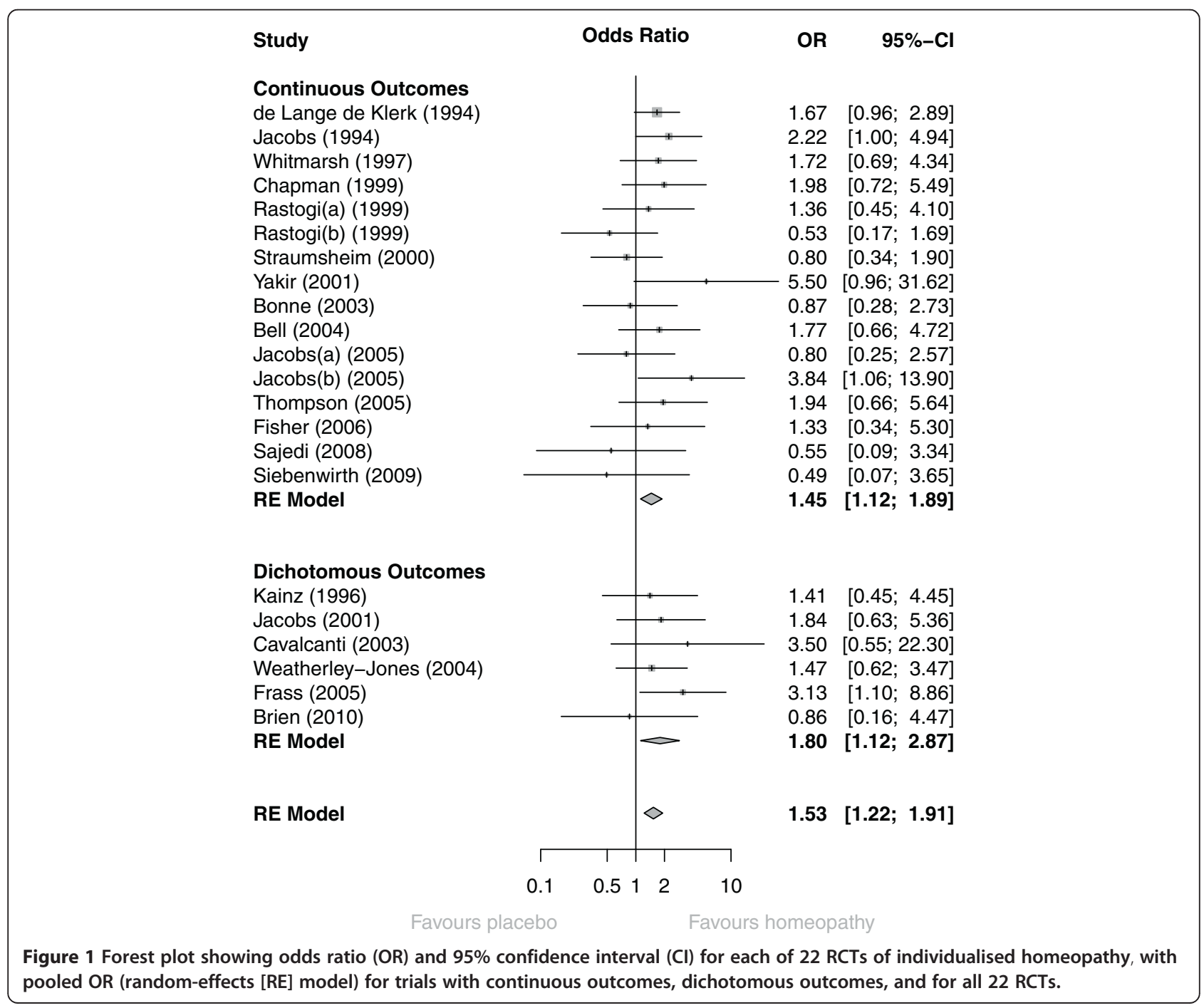




\section{Heterogeneity and publication bias}

Despite the clinical heterogeneity across the studies, the statistical heterogeneity between the studies was low $\left(I^{2}=\right.$ $0 \%$ [ $95 \%$ CI $0 \%$ to $40 \%]$ ), and therefore the variability in the estimated pooled OR is also relatively low. No evidence of publication bias was apparent from the funnel plot (Figure 2) or from Egger's test $(P=0.59)$.

\section{Risk of bias and reliable evidence}

Figure 3a shows the OR data for all 22 trials, grouped by their ' $\mathrm{B}$ '- or ' $\mathrm{C}$ '-rating. There was no difference between ' $\mathrm{B}$ '- and ' $\mathrm{C}$ '-rated trials: $P=0.41$.

- 'Uncertain risk of bias' (all 'B'-rated; $N=12$ ): $\mathrm{OR}=1.63$ (95\% CI 1.24 to 2.14; $P<0.001)$;

- 'High risk of bias' (all 'C'-rated; $N=10$ ): $\mathrm{OR}=1.33$ (95\% CI 0.90 to $1.98 ; P=0.15$ ).

Figure $3 \mathrm{~b}$ shows the OR data for the 12 ' $\mathrm{B}$ '-rated trials, grouped by reliability of evidence. There was no difference between the two sub-sets $(P=0.42)$.

- Uncertain risk of bias/reliable evidence $(N=3)$ : $\mathrm{OR}=1.98$ (95\% CI 1.16 to $3.38 ; P=0.013)$.
- Uncertain risk of bias/non-reliable evidence $(N=9)$ : $\mathrm{OR}=1.53$ (95\% CI 1.09 to $2.14 ; P=0.014)$.

\section{Sensitivity analysis}

Figure 4 shows the effect of removing data by trials' risk-of-bias rating; full details are given in Additional file 8 . The set of six trials rated ' $\mathrm{B} 1$ ' has been subdivided by those with/without reliable evidence. The pooled OR showed a statistically significant effect in favour of homeopathy for every value of $N$, down to and including the final $N=3$ trials with reliable evidence. Additional file 8 also states whether a trial was included in the 'global' analyses by Linde and/or Shang [6,8]: of the $22 \mathrm{RCTs}$ we subjected to metaanalysis, 8 had previously been analysed and 14 had not (our selected 'main outcome measure' differs for 3 of the 8 trials also included by Shang-Additional file 9).

\section{Sub-group analyses}

Pooled OR favoured homeopathy for all sub-groups and was statistically significant for all but two of them ( $<$ median; potency not exclusively $\geq 12 \mathrm{C}$ ): Figure 5a. There was no evidence of difference in the pooled

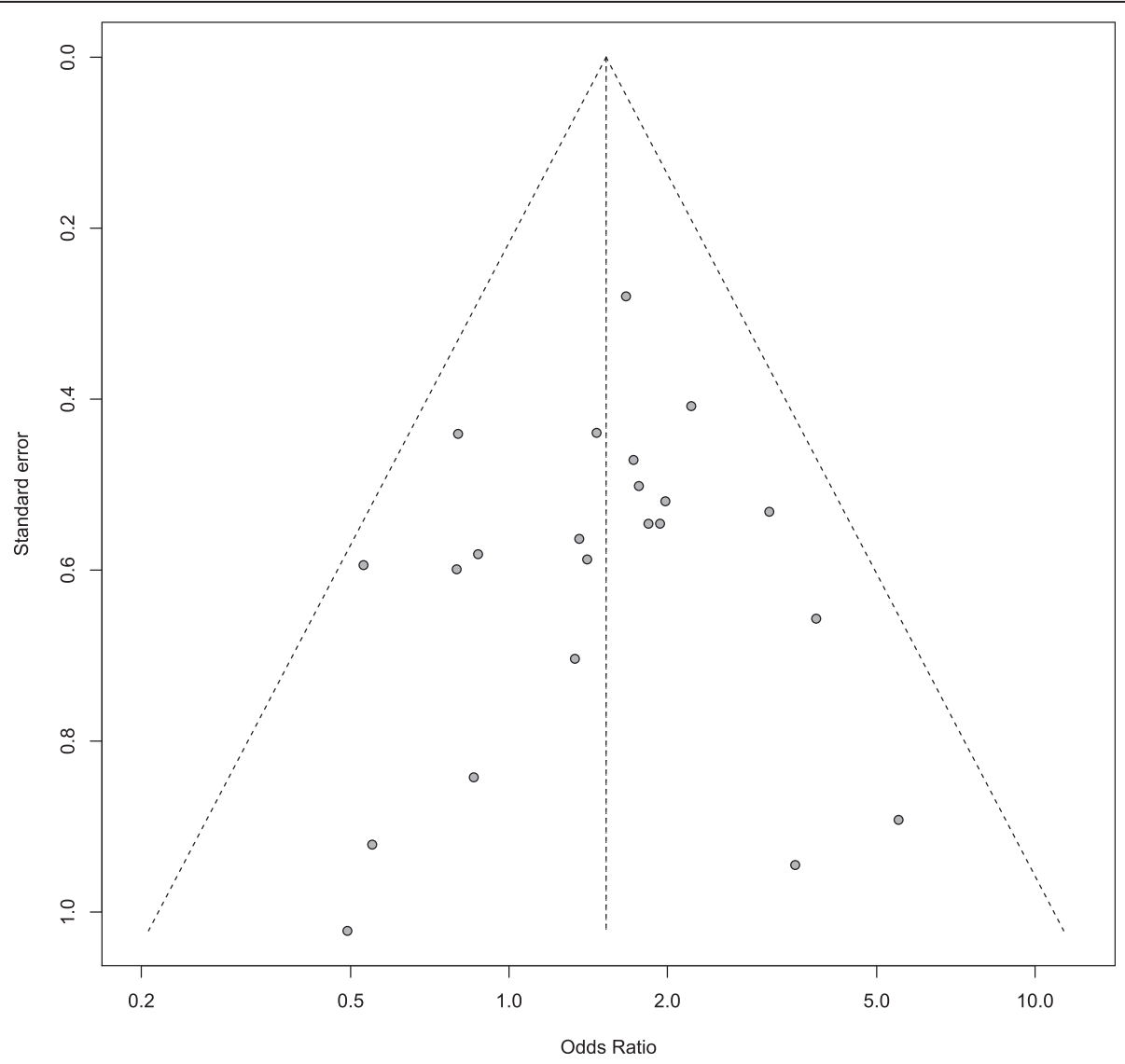

Figure 2 Funnel plot: 22 RCTs of individualised homeopathy. 


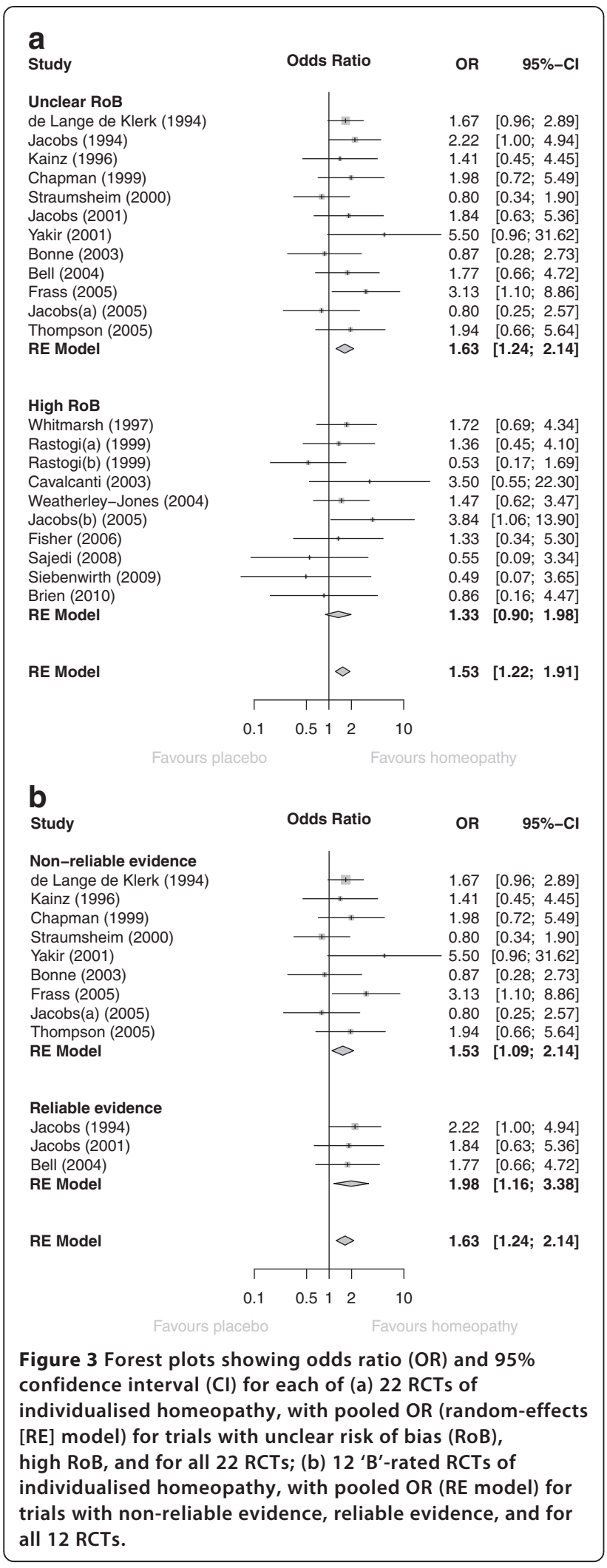

statistic between any sub-groups. Similar results were seen for the $N=12$ (Figure $5 \mathrm{~b}$ ) and $N=3$ analyses (data not illustrated). Full details are given in Additional file 10 . We observed a statistically significant pooled OR, favouring homeopathy, for the eight trials that we have in common with those previously reported by Shang et al. [8].

\section{Discussion}

Twenty-nine of the 32 trials had unclear or high risk of bias in important domains of assessment. Poor reporting or other deficiencies in the original papers prevented data extraction for meta-analysis from 10 of the 32 trials; the potential influence of the 10 on our overall meta-analysis is unknown, but because of their intrinsic low quality, their absence does not alter our principal conclusions. High and unclear risk of bias featured almost equally in our 22-trial analysis; thus, the overall quality of analysed evidence was low or unclear, necessitating caution in interpreting the findings.

As was the case for the previous 'global' systematic reviews of homeopathy RCTs that have included metaanalysis $[6,8]$, there are obvious limitations in pooling data from diverse medical conditions, outcome measures and end-points. Thus, a given pooled effects estimate here does not have a clear numerical meaning or relative clinical value: it is a summary measure that enables statistical significance and mean 'effect size' to be attributed and to be interpreted in testing an hypothesis that individually prescribed homeopathic medicines have specific effects.

Though our conclusions can be made most securely from three trials with reliable evidence, this sub-set of studies is too small to enable a decisive answer to our tested hypothesis. Equivocal RCT evidence of this nature is not unusual in medical science, in which conclusions are commonly based on just two eligible RCTs per systematic review [17]. Given the specific focus of our study, a statistically significant OR of 1.98 may be interpreted as a small 'effect size' for these three trials collectively and does not differ significantly from the 'effect size' observed in our analysis of 22 trials $(\mathrm{OR}=1.53)$. Such 'effect sizes' seem comparable with, for example, sumatriptan for migraine, fluoxetine for major depressive disorder and cholinesterase inhibitors for dementia [18]. The detection of a small yet significant pooled OR, with the perspective that only a few single trials showed statistically significant effects, supports conjecture that the impact of an individualised homeopathic prescription may be difficult to observe readily in the context of any one particular placebo-controlled trial $[19,20]$. 
Our approach to quality assessment was objective and internally consistent, and the methodological implications of our risk-of-bias findings are the same as those for RCTs in conventional medicine. Indeed, it is noteworthy that domain II (allocation concealment) had the fewest 'low risk of bias' assessments (for 31\% of our trials): this compares historically with adequately described allocation concealment in just $16 \%$ conventional medicine trials that were published during the period 1960 to 1995 [21].

Two of the three trials with reliable evidence used medicines that were diluted beyond the Avogadro limit. Our pooled effects estimate for the three trials, therefore, is either a false positive or it reflects the relevance of new hypotheses about the biological mechanism of action of homeopathic dilutions [22,23]. It should also be noted that one of these same three trials displayed evidence of vested interest. It remains to be seen if our assessments of model validity [24] support or refute the legitimacy of these three trials as currently the most important contributors to the evidence base in individualised homeopathy.

\section{Comparison with previous systematic reviews}

Our database is different from that of both Linde and Shang $[6,8]$. Firstly, we concentrated solely on peerreviewed trials of individualised homeopathic treatment. Secondly, we have an updated set of trials for meta-analysis: 14 of our 22 RCTs were not included in the previous 'global' analyses. Thirdly, our selected 'main outcome measure'-and thus our calculated OR-differs for three of the eight previously analysed trials of individualised homeopathy. Fourthly, our group of three RCTs with 'reliable evidence' is founded on a more exacting standard than for Shang's 'trials of higher methodological quality': indeed, by Shang's explicit criteria for domains IV, V and VI, we would label five of our 'non-reliable' trials [20,25-28] as 'higher methodological quality'.

A notable finding from sub-group analysis is that our 14 newly examined trials do not differ in 'effect size' from the eight that were included in previous 'global' meta-analysis by Shang et al. [8], disputing suggestions that the evidence base in homeopathy is weakening with time [29]. Noteworthy too is the significantly positive pooled OR that we observed for those eight trials and the close similarity of its value to that calculable from Shang's forest plot data for the same eight [30].

Like us, Linde and colleagues reported a pooled OR of 1.50-2.00 for the highest-quality RCTs [3,6]. In their separate analysis of individualised homeopathy, however, Linde and Melchart noted a smaller 'effect size' whose statistical significance was only marginally not sustained for the highest-quality trials [9]. Unlike our predecessors, we found no evidence that lowerquality trials displayed a larger treatment effect than that of higher-quality studies: indeed, our ten ' $C$ 'rated trials with extractable data displayed a nonsignificant pooled effects estimate. It may be that our stringent judgmental approach led to a less extreme range of quality assessments than those of the earlier reviewers. Importantly, we found no evidence of publication bias, removing any need for data adjustment. 


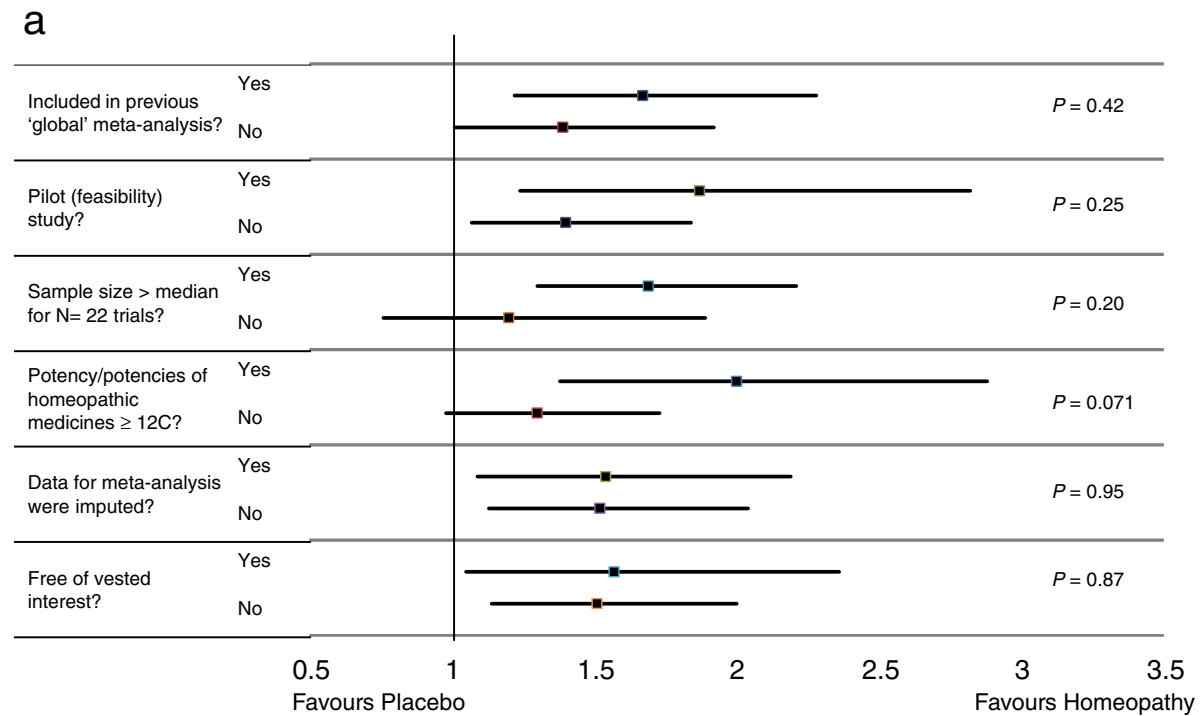

Odds Ratio: Homeopathy vs. Placebo (95\% Confidence Interval)

b

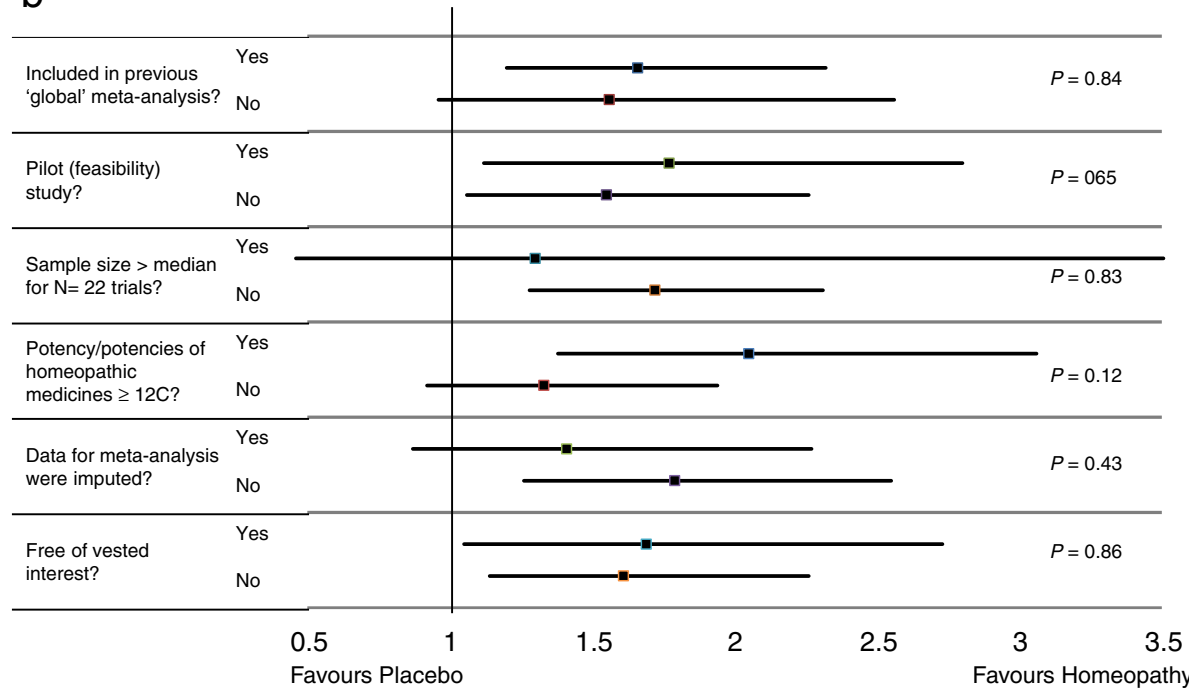

Odds Ratio: Homeopathy vs. Placebo (95\% Confidence Interval)

Figure 5 Interactions between sub-groups for (a) all $N=22$ trials with analysable data and (b) $N=12$ ' $\mathrm{B}^{\prime}$-rated trials.

\section{Conclusions}

- There was a small, statistically significant, treatment effect of individualised homeopathic treatment that was robust to sensitivity analysis based on 'reliable evidence'.

- Findings are consistent with sub-group data available in a previous 'global' systematic review of homeopathy RCTs.

- The overall quality of the evidence was low or unclear, preventing decisive conclusions.

- New RCT research of high quality on individualised homeopathy is required to enhance the totality of reliable evidence and thus enable clearer interpretation and a more informed scientific debate.

\section{Additional files}

Additional file 1: Checklist. PRISMA 2009 Checklist.

Additional file 2: PRISMA flowchart for records published in 2012 or 2013.

Additional file 3: PRISMA flowchart for all records published up to and including 2013.

Additional file 4: Details of records of RCTs of individualised homeopathy. Asterisk: paper reports two RCTs. Double asterisk: trial 
reported as double-blinded, but revealed to be single-blinded on inspection of published protocol. SD standard deviation. Reference numbering continues from previously published listings [11].

\section{Additional file 5: Risk-of-bias bar-graph for 32 RCTs of individualised} homeopathy.

Additional file 6: Data extracted for meta-analysis of RCTs with: (a) continuous main outcome measure and (b) dichotomous main outcome measure.

Additional file 7: Standardised mean difference (SMD) and $95 \%$ confidence interval (CI) for RCTs with continuous main outcome measure, showing pooled statistic (random effects [RE] model).

Additional file 8: Sensitivity analysis on risk-of-bias rating, and including specified demographic data per trial.

Additional file 9: Meta-analysed trials in common with those included by Shang et al. [8], showing comparison of quality assessment and degree of similarity of selected outcome measure. Additional file 10: Sub-group analysis showing interaction for: (a) all $N=22$ trials with analysable data, (b) $N=12$ ('B'-rated) trials with uncertain risk of bias and (c) sub-set of ('B1'-rated) trials with reliable evidence.

\section{Abbreviations}

Cl: Confidence interval; OR: Odds ratio; RCT: Randomised controlled trial; SMD: Standardised mean difference; WHO: World Health Organization.

\section{Competing interests}

RTM, JC and SM are either employed by or associated with a homeopathy charity to clarify and extend an evidence base in homeopathy. The University of Glasgow was supported by a grant from the British Homeopathic Association. All authors have applied the normal high standards of scientific method in the conduct of the work and of complete and transparent reporting in the write-up of the paper.

\section{Authors' contributions}

RTM devised and led the study, developed the study protocol and contributed to all facets of the work except the detailed statistical analysis. SML helped to develop the statistical protocol, conducted the statistical analyses and edited the manuscript. LAL helped to develop the study protocol, co-assessed trials for risk of bias, contributed to data interpretation and edited the manuscript. JC helped to develop the study protocol, co-assessed trials for risk of bias, contributed to data interpretation and edited the manuscript. SM co-assessed trials for risk of bias, contributed to data interpretation and edited the manuscript. JRTD helped to develop the study protocol, co-assessed trials for risk of bias, contributed to data interpretation and edited the manuscript. IF helped to develop the statistical protocol, contributed to data interpretation and edited the manuscript. All authors read and approved the final manuscript.

\section{Authors' information}

The study is intrinsic to the work of the British Homeopathic Association through its Research Development Adviser, RTM; it was assisted by a grant from the Manchester Homeopathic Clinic (see below). Trustees and staff of neither charity contributed to the design, analysis or write-up. RTM devised and led the study in collaboration with his co-authors.

\section{Acknowledgements}

The study was supported by a grant from the Manchester Homeopathic Clinic to the British Homeopathic Association.

\section{Author details}

Bitish Homeopathic Association, Luton, UK. ${ }^{2}$ Robertson Centre for Biostatistics, Institute of Health and Wellbeing, University of Glasgow, Glasgow, UK. ${ }^{3}$ Department of Biomedical Engineering, University of Strathclyde, Glasgow, UK. ${ }^{4}$ Karl und Veronica Carstens-Stiftung, Essen, Germany. ${ }^{5}$ Homeopathy Research Institute, London, UK. ${ }^{6}$ Department of Psychiatry and Behavioral Sciences, Duke University Medical Center, Durham, $N C$, USA.
Received: 26 June 2014 Accepted: 12 November 2014

Published: 6 December 2014

\section{References}

1. Vithoulkas G: Another point of view for the homeopathic trials and meta-analyses. [http://www.vithoulkas.com/en/research/articles/2247.html]

2. Sense About Science: Homeopathy. [http://www.senseaboutscience.org/ data/files/resources/54/Homeopathy.pdf.]

3. Linde K, Scholz M, Ramirez G, Clausius N, Melchart D, Jonas WB: Impact of study quality on outcome in placebo-controlled trials of homeopathy. J Clin Epidemiol 1999, 52:631-636.

4. Kleijnen J, Knipschild P, ter Riet G: Clinical trials of homoeopathy. BMJ 1991, 302:316-323.

5. Boissel JP, Cucherat M, Haugh M, Gauthier E: Critical literature review on the effectiveness of homoeopathy: overview of data from homoeopathic medicine trials. In Homoeopathic Medicine Research Group, Report of the Commission of the European Communities, Directorate-General XII - Science, Research and Development, Directorate E - RTD Actions. Brussels, Belgium: Life Sciences and Technologies - Medical Research; 1996.

6. Linde K, Clausius N, Ramirez G, Melchart D, Eitel F, Hedges LV, Jonas WB: Are the clinical effects of homoeopathy placebo effects? A meta-analysis of placebo-controlled trials. Lancet 1997, 350:834-843.

7. Cucherat M, Haugh MC, Gooch M, Boissel JP: Evidence of clinical efficacy of homeopathy. A meta-analysis of clinical trials. Eur J Clin Pharmacol 2000, 56:27-33.

8. Shang A, Huwiler-Muntener K, Nartey L, Juntherapiesi P, Dorig S, Sterne JA, Pewsner D, Egger M: Are the clinical effects of homoeopathy placebo effects? Comparative study of placebo-controlled trials of homoeopathy and allopathy. Lancet 2005, 366:726-732.

9. Linde K, Melchart D: Randomized controlled trials of individualized homeopathy: a state-of-the-art review. J Altern Complement Med 1998, 4:371-388.

10. Mathie RT, Legg LA, Clausen J, Davidson JRT, Lloyd SM, Ford I: Systematic review and meta-analysis of randomised, placebo-controlled, trials of individualised homeopathic treatment: study protocol. 2013, Version 1.0 25 January 2013. [http://www.britishhomeopathic.org/wp-content/uploads/ 2013/05/Study_protocol_for_systematic_review.pdf]

11. Mathie RT, Hacke D, Clausen J, Nicolai T, Riley DS, Fisher P: Randomised controlled trials of homeopathy in humans: characterising the research journal literature for systematic review. Homeopathy 2013, 102:3-24.

12. World Health Organization: Towards a Common Lanquage for Functioning, Disability and Health: The International Classification of Functioning, Disability and Health (ICF). Geneva: World Health Organization; 2002.

13. Higgins JPT, Altman DG: Chapter 8: Assessing risk of bias in included studies. In Cochrane Handbook for Systematic Reviews of Interventions, Version 5.1.0. Edited by Higgins JPT, Green S. The Cochrane Collaboration; 2011.

14. Sterne JAC, Egger M, Moher D: Chapter 10: Addressing reporting biases. In Cochrane Handbook for Systematic Reviews of Interventions; Version 5.1.0. Edited by Higgins JPT, Green S. The Cochrane Collaboration; 2011.

15. Egger M, Smith GD, Schneider M, Minder C: Bias in meta-analysis detected by a simple, graphical test. BMJ 1997, 315:629-634.

16. Bellavite P, Marzotto M, Olioso D, Moratti E, Conforti A: High-dilution effects revisited. 1. Physicochemical aspects. Homeopathy 2014, 103:4-21.

17. El Dib RP, Atallah AN, Andriolo RB: Mapping the Cochrane evidence for decision making in health care. J Eval Clin Pract 2007, 13:689-692.

18. Leucht S, Hierl S, Kissling W, Dold M, Davis JM: Putting the efficacy of psychiatric and general medicine medication into perspective: review of meta-analyses. Br J Psychiatry 2012, 200:97-106.

19. Weatherley-Jones E, Thompson EA, Thomas KJ: The placebo-controlled trial as a test of complementary and alternative medicine: observations from research experience of individualised homeopathic treatment. Homeopathy 2004, 93:186-189.

20. Brien S, Lachance L, Prescott P, McDermott C, Lewith G: Homeopathy has clinical benefits in rheumatoid arthritis patients that are attributable to the consultation process but not the homeopathic remedy: a randomized controlled clinical trial. Rheumatology (Oxford) 2011, 50:1070-1082

21. Moher D, Pham B, Jones A, Cook DJ, Jadad AR, Moher M, Tugwell P, Klassen TP: Does quality of reports of randomised trials affect estimates 
of intervention efficacy reported in meta-analyses? Lancet 1998, 352:609-613.

22. Bell IR, Koithan M: A model for homeopathic remedy effects: low dose nanoparticles, allostatic cross-adaptation, and time-dependent sensitization in a complex adaptive system. BMC Complement Altern Med 2012, 12:191.

23. Bellavite $\mathrm{P}$, Marzotto M, Olioso D, Moratti E, Conforti A: High-dilution effects revisited. 2. Pharmacodynamic mechanisms. Homeopathy 2014, 103:22-43.

24. Mathie RT, Roniger $H$, Van Wassenhoven $M$, Frye J, Jacobs J, Oberbaum M, Bordet M-F, Nayak C, Chaufferin G, Ives JA, Dantas F, Fisher P: Method for appraising model validity of randomised controlled trials of homeopathic treatment: multi-rater concordance study. BMC Med Res Methodol 2012, 12:49.

25. Jacobs J, Herman P, Heron K, Olsen S, Vaughters L: Homeopathy for menopausal symptoms in breast cancer survivors: a preliminary randomized controlled trial. J Altern Complement Med 2005, 11:21-27.

26. Jacobs J, Jimenez LM, Malthouse S, Chapman E, Crothers D, Masuk M, Jonas WB: Homeopathic treatment of acute childhood diarrhoea: results from a clinical trial in Nepal. J Altern Complement Med 2000, 6:131-139.

27. White A, Slade P, Hunt C, Hart A, Ernst E: Individualised homeopathy as an adjunct in the treatment of childhood asthma: a randomised placebo controlled trial. Thorax 2003, 58:317-321.

28. Steinsbekk A, Bentzen N, Fønnebø V, Lewith G: Self treatment with one of three self selected, ultramolecular homeopathic medicines for the prevention of upper respiratory tract infections in children. A double-blind randomized placebo controlled trial. Br J Clin Pharmacol 2005, 59:447-455.

29. Ernst $\mathrm{E}$, Pittler MH, Wider B, Boddy K: Homeopathy: is the evidence-base changing? Perfusion 2006, 19:380-382.

30. British Homeopathic Association: Data from randomised controlled trials of individualised homeopathy, reported by Shang et al. in 2005. [http://www.britishhomeopathic.org/wp-content/uploads/2014/11/Shang-reanalysis-individualised.pdf]

doi:10.1186/2046-4053-3-142

Cite this article as: Mathie et al:: Randomised placebo-controlled trials of individualised homeopathic treatment: systematic review and meta-analysis. Systematic Reviews 2014 3:142.

\section{Submit your next manuscript to BioMed Central and take full advantage of:}

- Convenient online submission

- Thorough peer review

- No space constraints or color figure charges

- Immediate publication on acceptance

- Inclusion in PubMed, CAS, Scopus and Google Scholar

- Research which is freely available for redistribution 